$16^{\text {th }}$ International Congress of Metrology, 17001 (2013)

DOI: $10.1051 /$ metrology/201317001

(C) Owned by the authors, published by EDP Sciences, 2013

\title{
TIME AS UNIQUE BASE QUANTITY
}

\author{
Gheorghe Dragan * \\ GDF DATA BANKS srl, str. Abrud 25, Bucharest 011315, ROMANIA, \\ www.gdfdatabanks.ro
}

\begin{abstract}
Main topoenergetic principles are reviewed in view to emphasize the UNIVERSAL relationships between time conversion of a system in transformation and one of its governing potentials. It results that: (i) any quantity can be calibrated in unit of time and (ii) any quantity can be calibrated by another one.
\end{abstract}

\section{Short history of topoenergetic principles}

Topoenergetic principles were initiated by calorimetric studies of polyethylene morphologies created by different thermal, mechanical and/or chemical treatments [1,2]. The basic observation was that all systems in transformation have composite structure because at least two components interact between them and exchange energy (heat) with the surrounding medium (Figure 1). Calorimetry was the most appropriate analytical technique allowing development of topoenergetic principles $[1,3]$. In view to quantitatively describe the kinetics of the transforming process by its components, I considered the energy circuit in similar terms like electric circuits, i.e. as composed by components with elementary behaviours: resistors, capacitors, energy sources, etc. This idea was launched in the same period of time by Oster and Auslander [4], but unfortunately they considered on the one hand very complicated cases and on the other hand that these components are spatially distributed in the transforming system. In this way mathematical description became unrealistic.

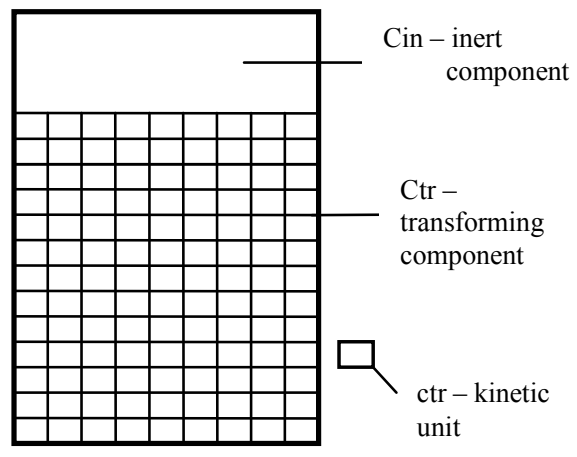

Figure 1. Composite structure of a test specimen in transformation.

The first kinetic equation established in topoenergetic terms was a modified Arrhenius equation applied for crystallization of polyethylenes by using a so called "drop calorimetry" [5]. The experimental principle of this technique consists in keeping first the calorimetric cell with the specimen at a temperature over melting point, $\mathrm{Tm}$, and dropping it in the calorimeter already prepared at crystallization temperature, T. Two exothermal flows appear, namely: one immediately at the contact with calorimeter (at time considered zero) given by inert component, Cin, denoted as win, and the flow delayed at a specific time, ti, given by component in transformation, Ctr (wtr), in this case the phase which crystallizes. The kinetic equation is:

$$
\ln (\mathrm{ti} * \mathrm{~T})=-\mathrm{E} /\left(R^{*} \mathrm{~T}\right)+\mathrm{K}
$$

where $\mathrm{E}$ - activation energy, $R$ - the gas constant, $\mathrm{T}$ in Kelvin and the free term

$$
\mathrm{K}=\ln (\operatorname{Cin} * \mathbf{R} * \mathrm{E} / R)
$$

\footnotetext{
* Corresponding author: dragan_gdf@yahoo.com,gdf.dragan@gmail.com
} 
where $\mathbf{R}$ - is the dissipative (resistive) element specific to the experimental device. It is important to mention that if we repeat experiments with the same sample, but using different specimen size and/or calorimeters, the parameters (ti, E, K) may be different. In this experiment temperature is the potential governing the transforming process and $\mathrm{Tm}$ is the threshold value above which the crystallization does not occur. In other words, the calorimetric experiment reveals the evolution of the test specimen between two equilibrium states located on the both sides of the threshold value.

The same equation was proved to be valid for processes occurring by increasing temperature, i.e. the process occurs at a temperature over the threshold value (for instance melting [6], curing-polymerization [7], etc.). By considering more transforming processes driven by temperature by using different measuring systems (analytical techniques), but the same principle of stepwise perturbation crossing a threshold value, kinetic equation remains valid $[8,9]$.

Table 1. ARRHENIUS representation:

$$
\ln \left(\mathrm{ti}^{*} \mathrm{~T}\right)=-\mathrm{E} /\left(R^{*} \mathrm{~T}\right)+\mathrm{K}
$$$$
\mathrm{K}=\mathrm{n} 1 * \mathrm{E}+\mathrm{m} 1
$$

\begin{tabular}{|c|c|c|c|}
\hline win*wtr & E*n1 & P & E \\
\hline+ & + & + & $\ln (\mathrm{Ctr})$ \\
\hline- & - & - & $-\ln (\mathrm{Ctr})$ \\
\hline
\end{tabular}

However, Arrhenius representation is very limited firstly because kinetic equation (1) does not consider threshold value (generally denoted as To), and secondly because can not describe the all kinds of transforming processes governed by general potential $U$.

By considering a large variety of transforming processes evidenced by the same experimental principle of stepwise perturbation crossing the threshold value Uo, the following UNIVERSAL kinetic equation was established:

$$
\ln (\mathrm{ti})=\mathrm{N}^{*} \ln |\mathrm{U}-\mathrm{Uo}|+\mathrm{M}
$$

where parameters (N,M,Uo) univocally define the behaviour of the tested sample in the considered measuring system. Parameters (E, K) and (N,M,Uo) define the amplitude and the nature of the transforming process or the ontogeny of the tested sample. Furthermore, if we test in the same measuring system different samples showing the same process of transformation, but with different ratio of $\mathrm{Cin}$ and $\mathrm{Ctr}$, their ontogeny parameters show the first phylogeny relationship in both Arrhenius and UNIVERSAL representations:

$$
\begin{aligned}
& \mathrm{K}=\mathrm{n} 1 * \mathrm{E}+\mathrm{m} 1 \\
& \mathrm{M}=\mathrm{n} 1 * \mathrm{~N}+\mathrm{m} 1
\end{aligned}
$$

where $(\mathrm{n} 1, \mathrm{~m} 1)$ are first phylogeny parameters defining the nature of the transforming process common to the tested samples.

Table 2. UNIVERSAL representation:

$$
\begin{gathered}
\ln (\mathrm{ti})=\mathrm{N}^{*} \ln |\mathrm{U}-\mathrm{Uo}|+\mathrm{M} \\
\mathrm{M}=\mathrm{n} 1 * \mathrm{~N}+\mathrm{m} 1
\end{gathered}
$$

\begin{tabular}{|c|c|c|c|c|c|}
\hline $\mathrm{N}^{*} \mathrm{n} 1$ & $\mathrm{~N}$ & $\mathrm{P}$ & $\mathrm{M}$ & $-\mathrm{M} / \mathrm{N}$ & $-\mathrm{N}^{\wedge} 2 / \mathrm{M}$ \\
\hline+ & $-\ln (\mathrm{Ctr})$ & - & $\ln (\mathrm{Ctr})$ & $\ln (\mathrm{ctr})$ & $\mathrm{CS}$ \\
\hline- & $\ln (\mathrm{Ctr})$ & + & $-\ln (\mathrm{Ctr})$ & $-\ln (\mathrm{ctr})$ & $-\mathrm{CS}$ \\
\hline
\end{tabular}

Another important parameter is the process polarity, $\mathrm{P}$, which was initially defined as a trivial parameter for thermally driven processes as revealed in calorimetric systems (Table 1).

UNIVERSAL representation allows defining also the coupling strength (CS) between Ctr and Cin (Table 2). For groups of samples showing different nature of transforming processes, it is possible to establish higher phylogenies [1,6], so the overall experimental data have a pyramidal structure (Table 3). For instance energy structure of atoms and all atomic spectra can be described by a unique pyramid of topoenergetic parameters [10].

\section{Standard Experimental Conditions (SEC)}

Figure 2 suggestively presents the experimental procedure in view to define the ontogeny of a system according to topoenergetic principles. There are represented three sections: the bellow sections represent the stepwise perturbation on the governing potential $U$ by increasing (1) or decreasing (2) crossing the threshold value Uo. In the upper section the triggered process of transformation is revealed by the time conversion of a response function, $\Theta$, between the two equilibrium values $\Theta 1,2$, or by its derivative, $\mathrm{d} \Theta / \mathrm{dt}$, defining the induction time, ti as the eigenvalue from the considered response function. In general the effect of Cin can be removed and/or neglected. It is important to mention that in view to define the ontogeny parameters and subsequently the higher phylogenies, all experiments must be performed in the same Standard Experimental Conditions (SEC), namely:

(i) Conditions of repeatability;

(ii) the same size \& shape of tested specimens;

(iii) by applying an increasing or decreasing stepwise perturbation of governing potential, U, crossing the threshold value Uo specific to the transforming process;

(iv) by considering the same response function, its eigenvalue, governing potential and by keeping unchanged the units for all quantities during all measurements;

(v) linear relationships can be established between parameters obtained in two different repeatable conditions [11]. 


\section{Metrological aspects}

The UNIVERSAL kinetic equation establishes the univocal relationship between ti and $U$ specific for a transforming process which can be considered as a calibration curve of $U$ units in time units.

Furthermore, ti is a particular case of eigenvalue from time conversion of the response function. It can be replaced by other specific quantities; for instance, electric resistance of NTC-thermistors as a function of temperature, a.c. electric conductivity quantities as a function of solute concentration in electrolyte solutions, etc. [10]. Topoenergetic principles can solve many problems of actual metrology (for instance viscosity).

Table 3. Pyramidal structure of data bank obtained according to topoenergetic principles.

\begin{tabular}{|l|l|}
\hline (ni, mi) & higher phylogeny parameters \\
\hline & \\
\hline$(\mathrm{n} 1, \mathrm{~m} 1)$ & first phylogeny parameters \\
\hline$(\mathrm{E}, \mathrm{K}),(\mathrm{N}, \mathrm{M}, \mathrm{Uo})$ & ontogeny parameters \\
\hline$(\mathrm{ti}, \mathrm{U})$ & $\begin{array}{l}\text { basic experimental data } \\
\text { obtained in accurate SEC }\end{array}$ \\
\hline
\end{tabular}

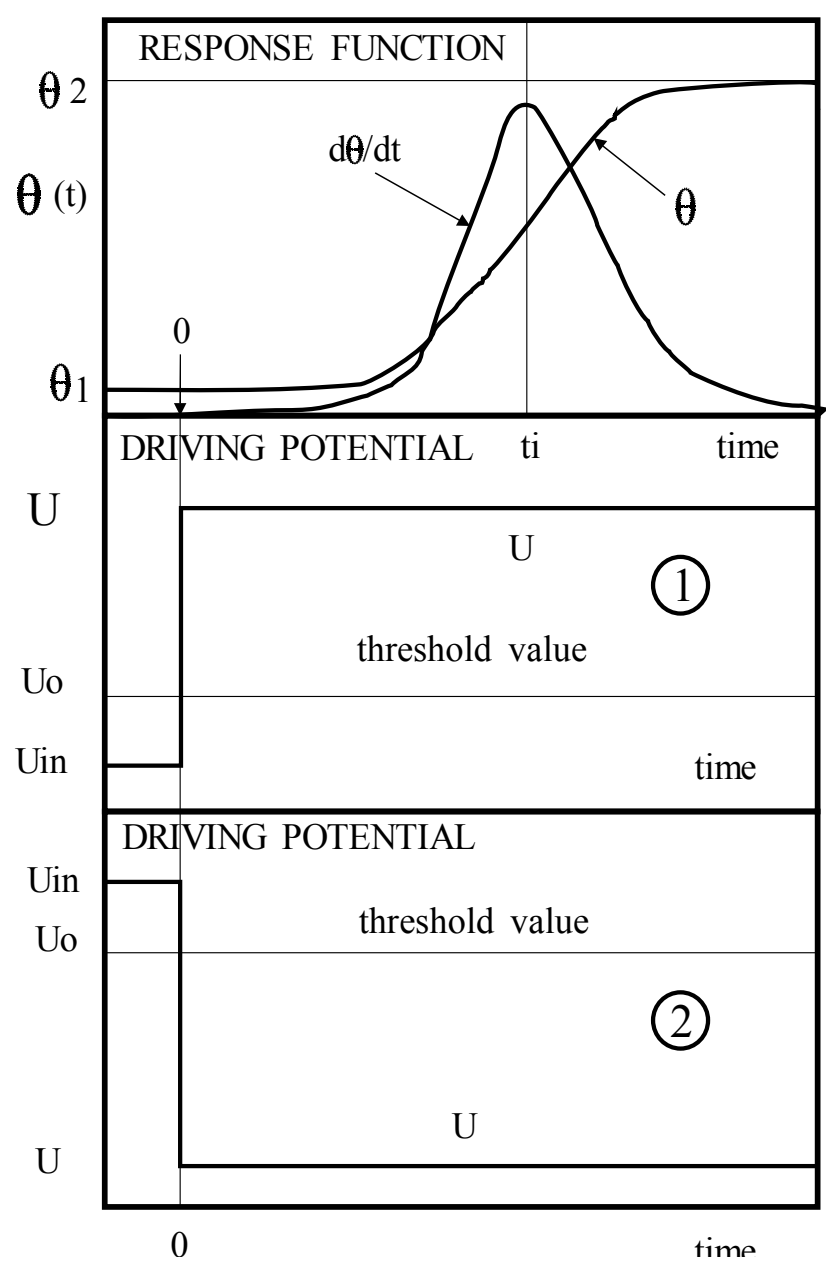

\section{References}

[1] Gh.Dragan, Rev. Roumaine Chim., 28(9,10), 1315 (1981).

[2] Gh.Dragan, J. Polymer Symposium, 64, 141 (1978).

[3] Gh.Dragan, J. Thermal Anal., 9, 405 (1978).

[4] G.F.Oster and D.M.Auslander, J. Franklin Inst., 292, 1(1971).

[5] Gh.Dragan, Rev. Roumaine Chim., 23(4), 629 (1978).

[6] Gh.Dragan, Acta Polymerica, 37(10), 620 (1966).

[7] Gh.Dragan and F.Stoenescu, Rev. Roumaine Chim., 24(1), 55 (1979).

[8] Gh.Dragan, and G.Banica, Phys.Chem. Mechanics, 10, 41 (1983).

[9] Gh.Dragan, GDF Databanks Bull., 15 (2), 3 (2011).

[10] GDF Databanks Bull. (ISSN 1453-1674) publishes applications of topoenergetic principles. It is registered at Biblioteca Nationala a Romaniei and National Library of Australia and it is also posted on the website www.gdfdatabanks.ro

[11] Gh.Dragan, Acta Polymerica, 36(9), 499 (1985).

Figure 2. 